\title{
Prevalence on the Types of Denture Stomatitis Reported in A Private Dental Hospital: A Retrospective Study
}

\author{
Mukundh Chaithanya V ${ }^{1}$, Uma Maheswari T. ${ }^{2 *}$ and Manjari Chaudhary ${ }^{2}$ \\ ${ }^{1}$ Saveetha Dental College and Hospitals, Saveetha Institute of Medical \\ and Technical Sciences (SIMATS), Chennai, India \\ ${ }^{2}$ Department of Oral Medicine and Radiology,Saveetha Dental College and Hospitals, \\ Saveetha Institute of Medical and Technical Sciences (SIMATS),Saveetha University, Chennai, India \\ Corresponding author e-mail: umamaheswaritn@saveetha.com
}

\begin{abstract}
Denture stomatitis affects the denture bearing surfaces of the oral mucosa, which is characterised by inflammation followed by erythema, denture stomatitis occurs due to superficial infection by candida species which is due to various factors such as ill-fitting prosthesis, long period of denture wearing. Predisposing factors such as diabetes mellitus, bad oral hygiene which in turn cause the accumulation of yeast organisms contribute towards denture stomatitis. The aim of this study was to find the prevalence of denture stomatitis and its association with oral hygiene status and diabetes mellitus. The results of this study showed that Type 2 denture stomatitis according to Newton's classification which is a localised area of erythema involving the whole or part of denture bearing surface was the most common type of denture stomatitis. Maxillary palatal surface was the most common site of occurrence of stomatitis, the drug which was most preferably used was topical $1 \%$ clotrimazole. Statistical analysis was done using Pearson chisquare analysis which revealed no statistically significant association between denture stomatitis and age, gender, oral hygiene status and diabetes mellitus. Association done between denture stomatitis and oral hygiene index-simplified has shown that there is no statistically significant association $(0.501 \mathrm{p}$ value $>0.05)$.
\end{abstract}

\section{KEY WORDS: DENTURE STOMATITIS; INFLAMMATION; NEWTON CLASSIFICATION; CANDIDIASIS}

\section{INTRODUCTION}

Denture stomatitis affects the denture bearing surfaces of the oral mucosa, it is characterised by areas of erythema which is preceded by inflammation in the mucosal area that is covered by denture. Epidemiology studies have shown that about 15$70 \%$ of denture wearers experienced denture stomatitis. The occurrence of denture stomatitis was due to the accumulation of yeast or organisms such as candida species which is due to various factors such as ill-fitting prosthesis, long period of denture wearing, older denture, ill-hygiene of the patient, continuous wearing of denture (Pattanaik et al. 2010; Gendreau and Loewy 2011; Steele et al. 2015; Aoun and Cassia, 2016; Dharman and Muthukrishnan 2016; Rohini

Biosc Biotech Res Comm P-ISSN: 0974-6455 E-ISSN: 2321-4007

\section{rossef}

Identifiers and Pagination

Year: 2021 Vol: 14 No (10) Special Issue

Pages: $31-37$

This is an open access article under Creative

Commons License Attribn 4.0 Intl (CC-BY).

DOI: $h t t p: / / d x . d o i . o r g / 10.21786 / b b r c / 14.10 .6$ and Kumar 2017; Muthukrishnan and Kumar 2017; Rohini and Kumar 2017; Patil et al. 2018; ; Chaitanya et al. 2018; Maheswari et al. 2018; Gauch et al. 2018; Subha and Arvind 2019; Krishnaswamy et al. 2020).

The classification of denture stomatitis is based on the classification given by newton in 1962: Type-1- a localised simple inflammation or pinpoint hyperemia, Type-2-an erythematous or generalised simple type seen as more diffuse erythema involving a part or the entire denture covered mucosa, Type-3- a granular Type commonly involving the centre part of the hard palate and the alveolar ridges. Previous research done suggests that the females: male ratio was about 3:1 showing the higher predilection towards females, along with other predisposing factors such as nocturnal denture wearers, non-maintenance of oral hygiene, microbes, duration of denture usage, systemic conditions such as diabetes mellitus and various immunodeficiencies, dryness of mouth (Xerostomia), trauma due to occlusion, poor nutritional status of the patient, foods high in carbohydrates, tobacco habits such as smoking and tobacco chewing, allergic reaction of the body towards certain denture resin materials, plaque accumulation on 
the mucosal surface of the denture, followed by age of the patient (Choudhury et al. 2015; Muthukrishnan et al., 2016; Karthikeyan et al., 2016; Chaitanya et al. 2017; Krishnaswamy et al. 2020).

Candidiasis induced denture stomatitis is the most common, candida organisms that are known to induce denture stomatitis includes Candida tropicalis, Candida dubliniensis and Candida krusei. Previously our team has a rich experience in working on various research projects across multiple disciplines (Chaitanya et al. 2017; Varghese et al., 2019; Venu et al., 2019; Samuel et al. 2019; Mehta et al. 2019; Sharma et al. 2019; Sureshbabu et al. 2019; Gheena and Ezhilarasan 2019; Vignesh et al. 2019; Ke et al. 2019; Jain et al. 2019; Sathish and Karthick 2020; Samuel et al., 2020; Muthukrishnan et al. 2020; Krishnaswamy et al. 2020; Jose et al., 2020). Now the growing trend in this area motivated us to pursue this project. The aim of this study is to assess the prevalence of denture stomatitis in a hospital setting. The objectives of this study was to find the factors that cause denture stomatitis and to evaluate its association with age and gender along with predisposing factors such as oral hygiene and diabetes mellitus.

Figure 1: Pie chart representing the age distribution in patients with denture stomatitis, Prevalence percentage of denture stomatitis was highest in 30-60 years as 57.1\%(grey), followed by $34.7 \%$ (blue) in $>60$ years, and least prevalent as $8.2 \%$ (green) in $10-29$ years.

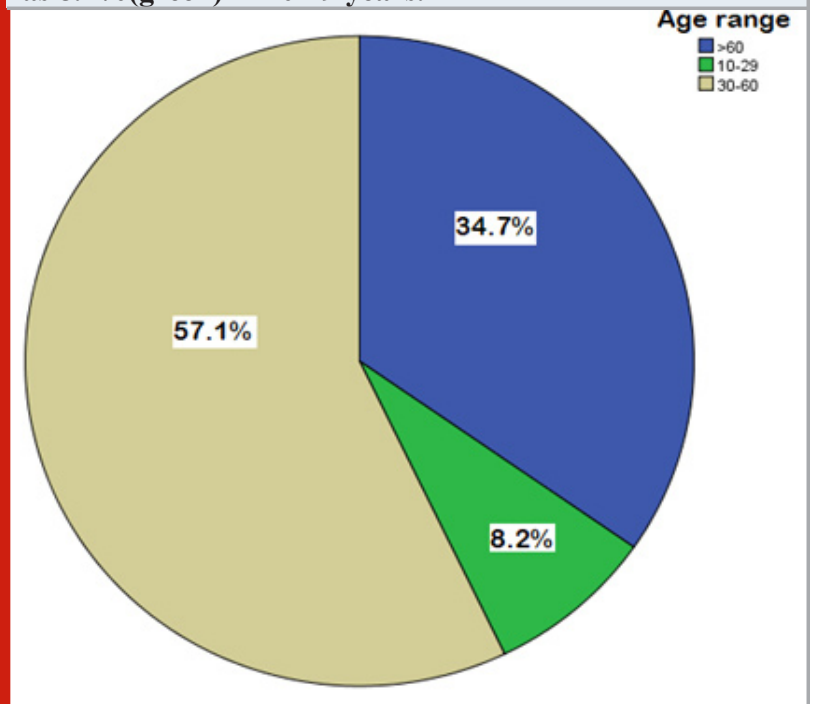

The lacunae of this study is less number of patients and statistical analysis of the present study is only of descriptive type, the rationale of this study to understanding the denture stomatitis and its age and gender based prevalence which would pay way for further epidemiological studies in the future.

\section{MATERIAL AND METHODS}

The retrospective study on prevalence of Aphthous stomatitis treated in the department of Oral Medicine was conducted in a hospital setting. Approval of ethical clearance was given by the Scientific Review Board SDC/SIHEC/2020/DIASDATA/0619-0320. The number of people involved in current research are three members (principal investigator, guide and reviewer). The sampling was done by collecting the data from September 2019 (01/09/2019) to April 2020 (01/04/2020). In this data, all the case sheets were reviewed, cross verification done, duplicate entries were removed and photographic evidence was used. The cases recorded with complete documentation of complaint, history of present illness and complete documentation of presence or absence of medical history and follow up cases alone were included in the study. The incomplete documented records with no proper treatment and follow up details were excluded from the study. Measures taken to minimise sampling is by means of convenience sampling and by selecting cases that are diagnosed and treated only in the oral medicine department.

Figure 2: Bar graph representing gender distribution in patients with denture stomatitis, $\mathrm{X}$-axis represents gender, Y-axis represents the frequency percentage of patients with denture stomatitis. Frequency distribution shows that gender distribution in this study was more in female patients $(59.2 \%)$ followed by male patients $(\mathbf{4 0 . 8 \%})$.

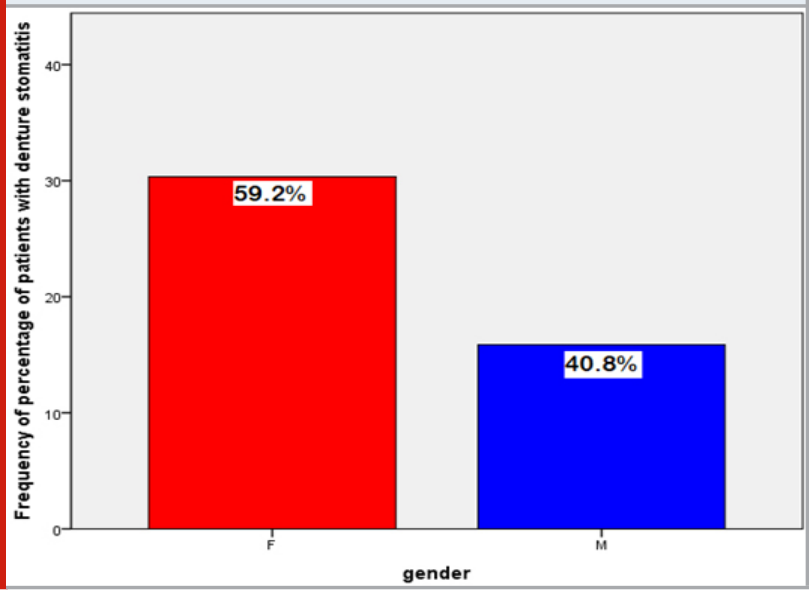

Figure 3: Bar graph representing frequency percentage of patients with denture stomatitis based on newton's classification of denture stomatitis. $X$-axis represents Newton's classification of patients with denture stomatitis, Y-axis represents the frequency percentage of patients with denture stomatitis and it revealed that Type-2(pink) as $\mathbf{5 9 . 2 \%}$, Type-1(orange) as $\mathbf{2 6 . 5 \%}$ and Type $\mathbf{3}$ (yellow) as $14.3 \%$ with highest prevalence reported in Type 2 denture stomatitis.

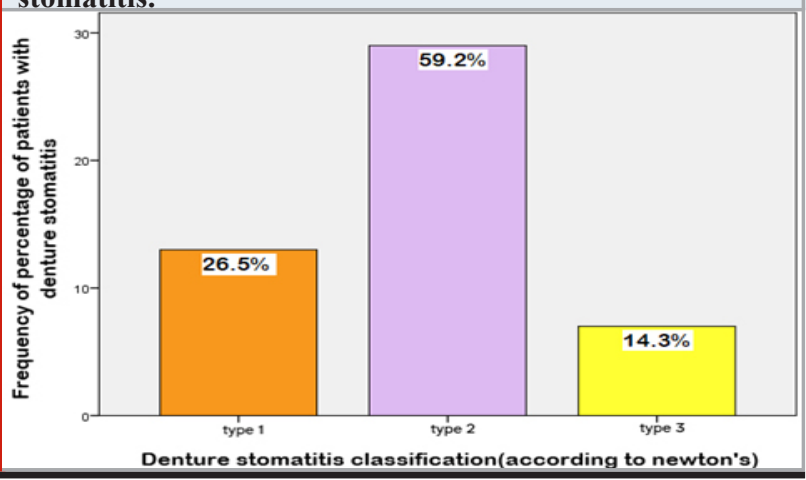


The current study was conducted in a hospital setting in which the demographic data such as such as age, gender and medical history data such as presence or absence of systemic diseases and general and oral examination details such as oral hygiene status and clinical features of denture stomatitis was retrieved from the database. The dependent variable considered as different types of denture stomatitis and independent variables were age, gender and oral hygiene status. The descriptive statistical tests used were frequency distribution tests. The inferential statistical tests such as the Pearsonchi square test were done. Statistical analysis such as frequency distribution and Pearson-chi square test was done using statistical software SPSS software (ver.26). Frequency distribution of types of denture stomatitis in age and gender and its association with age and gender, oral hygiene status and presence or absence of diabetes mellitus were analysed using Pearson chi-square analysis.

Figure 4: Bar graph representing the association between patients with denture stomatitis and age distribution, $\mathrm{X}$-axis represents the denture stomatitis classification, Y-axis represents number of patients with denture stomatitis. Association analysis between denture stomatitis and age was done using a Pearson chi-square test which revealed no statistically significant association $(0.434$, $p$ value $>0.05)$. However, Type 1,2 and 3 are most prevalent in the 30-60 years age group.

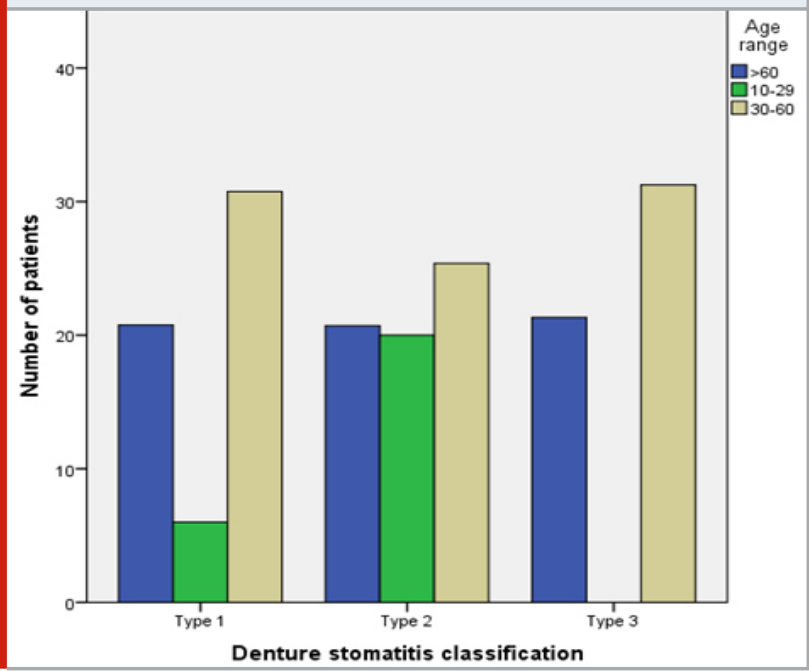

RESULTS AND DISCUSSION

This study has shown that Type-2 denture stomatitis according to Newton's classification most prevalent in about $80 \%$ of cases. The most common site of prevalence of denture stomatitis in about $80 \%$ of the cases was in palatal mucosa and remaining $20 \%$ in the alveolar ridge. The mean age of the patient was around 63 years. The prevalence of denture stomatitis was observed in 3 age groups. The percentage of prevalence in denture stomatitis was more in patients between 30-60 years $57.1 \%$ followed by, $34.7 \%$ in $>60$ years, and $8.2 \%$ in 10-29 years (Figure 1). Frequency analysis of gender distribution in patients with denture stomatitis shows that it was more prevalent in female patients, frequency analysis shows that gender predilection in this study was more towards female patients (59.2\%) followed by male patients (40.8\%) (Figure 2). The frequency percentage of patients with denture stomatitis based on Newton's classification has revealed that Type-2 type of denture stomatitis was more prevalent (59.2\%) followed by Type 1 in 26.5\% and Type 3 in 14.3\% (Figure 3).

Figure 5: Bar graph representing the association between patients with denture stomatitis and Gender, $\mathrm{X}$-axis represents the denture stomatitis classification, Y-axis represents number of patients with denture stomatitis. Pearson chi-square test revealed no statistically significant association $(\mathbf{0 . 3 5 2}, \mathrm{p}$ value $>\mathbf{0 . 0 5})$. However, it is seen that type $\mathbf{1}$ is more prevalent in males and type $\mathbf{3}$ in females.

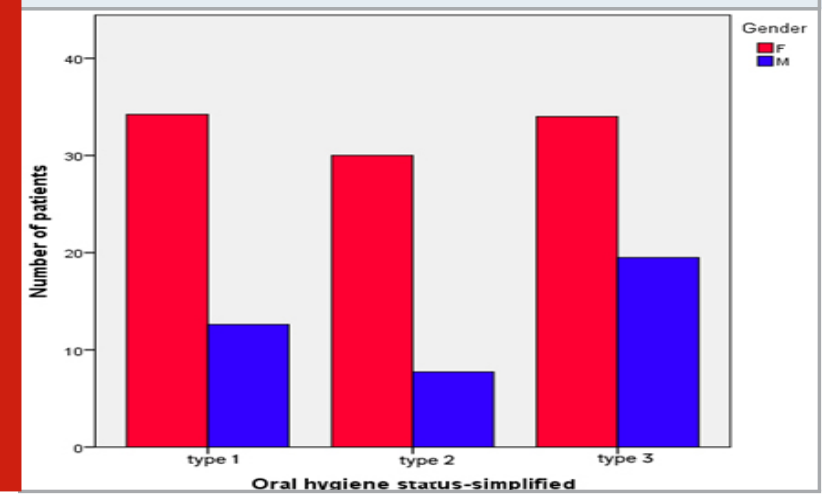

Figure 6: Bar graph representing the association between patients with denture stomatitis and $\mathrm{OHI}-\mathrm{S}, \mathrm{X}$-axis represents the denture stomatitis classification, Y-axis represents number of patients with denture stomatitis, Association analysis between denture stomatitis and OHI-S was done using pearson chi-square test which revealed no statistically significant association $(0.501$, $p$ value $>0.05)$. However poor oral hygiene was more prevalent in type 2 denture stomatitis patients.

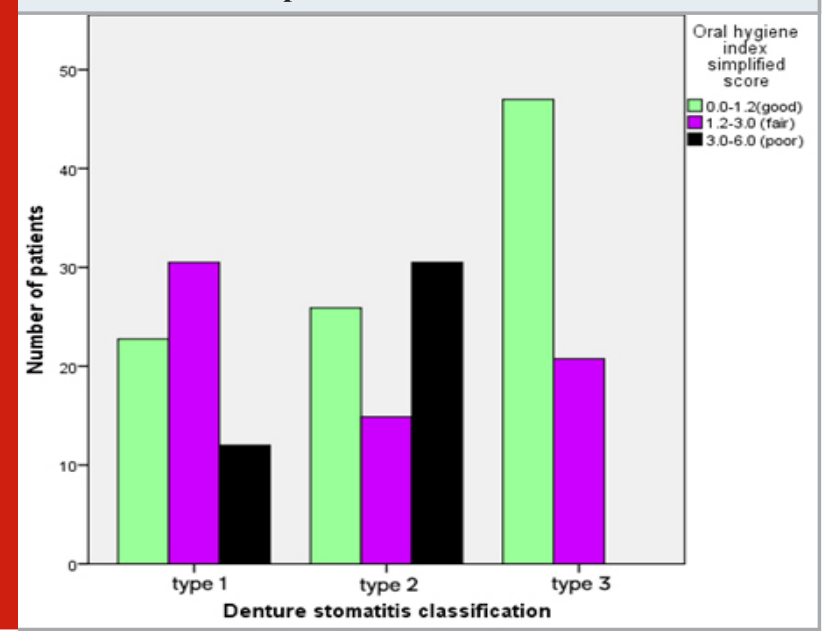

The association between denture stomatitis and age done has shown that denture stomatitis is more prevalent in the 30-60 years age group. Association analysis was 
done between denture stomatitis and age, using Pearson chi-square test which shows no statistical association (0.434, p value $>0.05)$. (Figure 4). The association between denture stomatitis and gender done shows that type 1 denture stomatitis is more prevalent in males and type 3 more prevalent in females. Association analysis between denture stomatitis and gender was done using a Pearson chi-square test which shows no statistically significant association (0.352, p value $>0.05$ ). (Figure 5). The association between denture stomatitis and Oral hygiene index-simplified (OHI-S) done shows that poor oral hygiene is more prevalent in type 2 denture stomatitis patients. Association analysis between denture stomatitis and $\mathrm{OHI}-\mathrm{S}$ was done using Pearson chi-square test which shows no statistically significant association (0.501, p value $>0.05)$. (Figure 6). The association between $\mathrm{OHI}-\mathrm{S}$ and age done shows that poor oral hygiene index is seen in patients in the $30-60$ years and 60 years above range.

Figure 7: Bar graph representing the association between patients with $\mathrm{OHI}-\mathrm{S}$ and age distribution in patients with denture stomatitis, $\mathrm{X}$-axis represents the OHI-S, Y-axis represents number of patients with denture stomatitis, Association analysis between OHI-S and age was done using Pearson chi-square test which revealed no statistically significant association $(\mathbf{0 . 3 5 2}$, $p$ value $>0.05)$. However, poor oral hygiene index is seen in patients in the 30-60 years and 60 years above range.

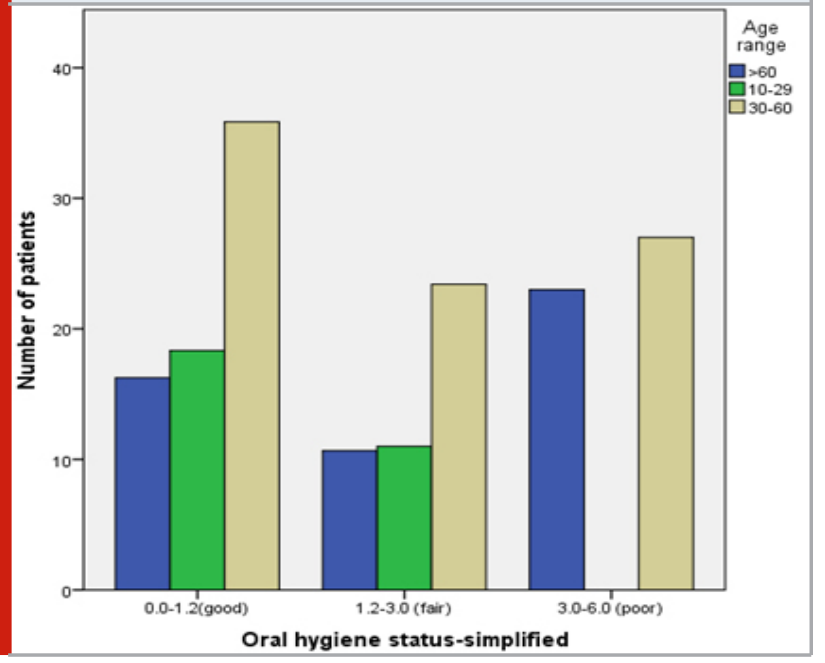

Association analysis between OHI-S and age was done using Pearson chi-square test which shows no statistically significant association (0.352, p value $>0.05$ ) (Figure 7). The drug of choice in the present study was $1 \%$ clotrimazole mouth paint which is used on all patients, the medical history that was more prevalent was diabetes mellitus, mean score of oral hygiene status of all the patients was about 1.6 (Fair). Frequency analysis of age distribution in patients with denture stomatitis reveals that denture stomatitis was more prevalent between the 30-60 years age group. The association between $\mathrm{OHI}-\mathrm{S}$ and gender done shows that poor hygiene status was more prevalent in male patients, both poor and good grades were prevalent in female patients. Association analysis between $\mathrm{OHI}-\mathrm{S}$ and gender was done using pearson chi-square test which shows no statistically significant association $(0.434$, p value $>0.05)$ (Figure 8).

The association between denture stomatitis and diabetes mellitus showed that Type 1 denture mellitus is more prevalent in diabetes mellitus patients while not prevalent in Type 2 and Type 3 patients. Association analysis between Denture stomatitis classification and diabetes mellitus was done using Pearson chi-square test which shows no statistically significant association (0.354, p value >0.05). However (Figure 9) Studies done by Pesee et al. (2015) has shown that gingivitis and diabetes has a strong association with the occurrence of denture stomatitis, the cause of xerostomia due to decreased salivary flow has resulted in less lubrication and more friction to the denture bearing surfaces and hence more chances of candida infection which is similar to the current study where diabetes is a predisposing factor for denture stomatitis (Pesee and Arpornsuwan 2015; Misra et al. 2015 Arvind et al., 2018; Mathew et al. 2020).

Figure 8: Bar graph representing the association between patients with $\mathrm{OHI}-\mathrm{S}$ and gender distribution in patients with denture stomatitis, $\mathrm{X}$-axis represents the $\mathrm{OHI}-\mathrm{S}, \mathrm{Y}$-axis represents number of patients with denture stomatitis. Pearson chi-square test revealed no statistical association $(\mathbf{0 . 4 3 4}$, $\mathrm{p}$ value $>\mathbf{0 . 0 5})$. Poor hygiene status more prevalent in male patients, both poor and good scores were prevalent in female patients

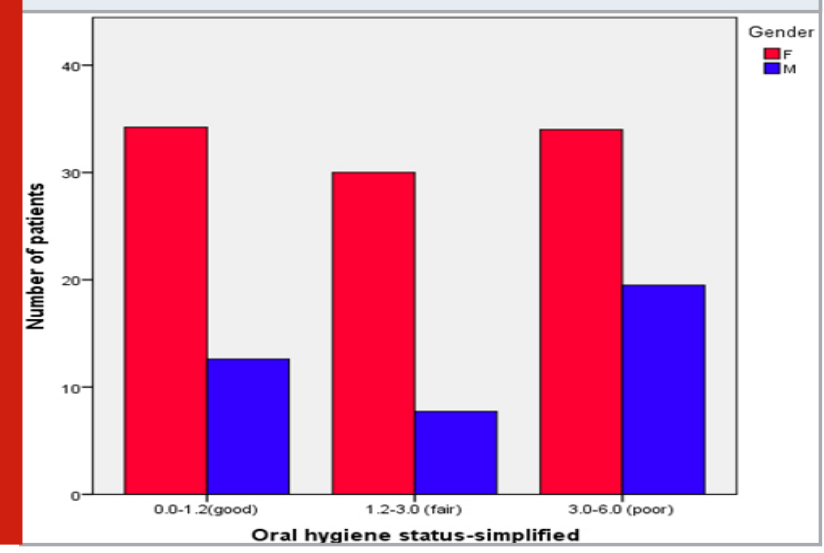

Few other studies revealed that denture stomatitis increases with age and medical conditions such as diabetes mellitus can be aggravating factors, along with predisposing factors such as the denture age/duration of denture wearing (in years/months) with oral hygiene scores of 2.3 (fair), but the present study has shown an $\mathrm{OHI}$ of 1.6(fair).Our institution is passionate about high quality evidence based research and has excelled in various fields Hyperlink "https://paperpile.com/c/ nAmPRM/hjrV5+Gzx5Z+LBvxT+sT3K1+9RbtK+x2JGP +m2eZ8" (Vijayashree Priyadharsini 2019; Ezhilarasan, Apoorva, and Ashok Vardhan 2019; Ramesh et al. 2018; 
Mathew et al. 2020; Sridharan et al. 2019; Pc, Marimuthu, and Devadoss 2018; Ramadurai et al. 2019). We hope this study adds to this rich legacy.

Figure 9: Bar graph representing the association between patients with denture stomatitis classification and history of diabetes mellitus. $\mathrm{X}$-axis represents the Denture stomatitis classification; Y-axis represents the number of patients with diabetes mellitus. Pearson chi-square test revealed no statistically significant association $(0.354, p$ value $>0.05)$. Diabetes mellitus was more prevalent in Type 1 denture stomatitis patients.

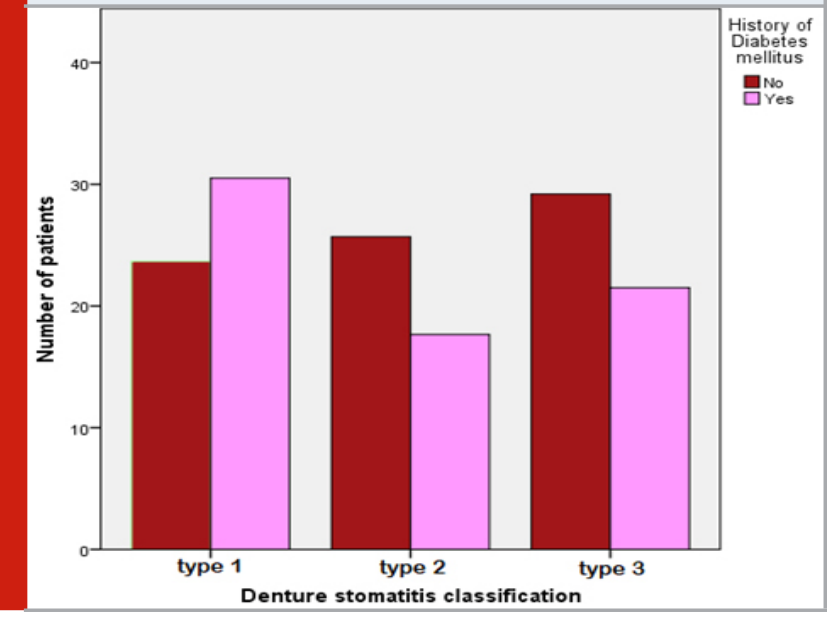

\section{CONCLUSION}

This study concludes that Type 2 denture stomatitis was the most prevalent in all age groups and patients with diabetes mellitus had more incidence of denture stomatitis. Topical application of 1\% clotrimazole has been successful in treating patients infected by denture stomatitis. The results of association between oral hygiene status and denture stomatitis has proved that there is no statistical significant association (0.501, p value $>0.05$ ), however the overall mean score of oral hygiene score was 1.6 in patients with denture stomatitis , hence this study results proves that maintenance of good oral hygiene plays a vital role in preventing recurrence of denture stomatitis especially with patients who are associated with other predisposing factors such as diabetes mellitus

\section{ACKNOWLEDGEMENTS}

Saveetha Dental College,Saveetha institute of medical and technical sciences

Conflicts of Interest: The authors declare that there were no conflicts of interest in the present study

\section{REFERENCES}

Aoun, Georges, and Antoine Cassia. (2016). Evaluation of Denture-Related Factors Predisposing to Denture Stomatitis in a Lebanese Population. Materia Socio Medica. https://doi.org/10.5455/msm.2016.28.392-396.
Chaitanya, Nallan Csk, Arvind Muthukrishnan, Dara Balaji Gandhi Babu, Chinta Sanjeeva Kumari, Madishetty Adi Lakshmi, Gayathri Palat, and Khwaja Shoeb Alam. (2017). Role of Vitamin E and Vitamin A in Oral Mucositis Induced by Cancer Chemo/Radiotherapy- A Meta-Analysis. Journal of Clinical and Diagnostic Research: JCDR 11 (5): ZE06-09.

Chaitanya, Nallan Csk, Arvind Muthukrishnan, C. M. S. Krishnaprasad, Gali Sanjuprasanna, Poojaragini Pillay, and Balmoori Mounika. (2018). An Insight and Update on the Analgesic Properties of Vitamin C. Journal of Pharmacy \& Bioallied Sciences 10 (3): 119-25.

Choudhury, Priyadarshini, Rajat G. Panigrahi, Maragathavalli, Antarmayee Panigrahi, and Padma Charan Patra. (2015). Vanishing Roots: First Case Report of Idiopathic Multiple Cervico-Apical External Root Resorption. Journal of Clinical and Diagnostic Research: JCDR 9 (3): ZD17-19.

Dharman, Sreedevi, and Arvind Muthukrishnan. (2016). Oral Mucous Membrane Pemphigoid - Two Case Reports with Varied Clinical Presentation. Journal of Indian Society of Periodontology 20 (6): 630-34.

Ezhilarasan, Devaraj, Velluru S. Apoorva, and Nandhigam Ashok Vardhan. (2019). Syzygium cumini Extract Induced Reactive Oxygen Species-Mediated Apoptosis in Human Oral Squamous Carcinoma Cells. Journal of Oral Pathology \& Medicine: Official Publication of the International Association of Oral Pathologists and the American Academy of Oral Pathology 48 (2): 115-21.

Gauch, Lurdete Maria Rocha, Simone Soares Pedrosa, Fabíola Silveira-Gomes, Renata Antunes Esteves, and Silvia Helena Marques-da-Silva. (2018). Isolation of Candida Spp. from Denture-Related Stomatitis in Pará, Brazil. Brazilian Journal of Microbiology: publication of the Brazilian Society for Microbiology 49 (1): 148-51.

Gendreau, Linda, and Zvi G. Loewy. (2011). Epidemiology and Etiology of Denture Stomatitis. Journal of Prosthodontics: Official Journal of the American College of Prosthodontists 20 (4): 251-60.

Gheena, S., and D. Ezhilarasan. (2019). Syringic Acid Triggers Reactive Oxygen Species-Mediated Cytotoxicity in HepG2 Cells. Human \& Experimental Toxicology 38 (6): 694-702.

Jose, Jerry, Ajitha, and Haripriya Subbaiyan. (2020). Different Treatment Modalities Followed by Dental Practitioners for Ellis Class 2 Fracture - A QuestionnaireBased Survey. The Open Dentistry Journal 14 (1): 59-65.

Karthikeyan, S., F. Teny, and P. V. Deepthi. (2016). Denture Stomatitis: A Brief Review. Journal of Medical and Dental Sciences 15: 114-16.

Ke, Yang, Mohammed Saleh Al Aboody, Wael Alturaiki, Suliman A. Alsagaby, Faiz Abdulaziz Alfaiz, Vishnu 
Priya Veeraraghavan, and Suresh Mickymaray. (2019). Photosynthesized Gold Nanoparticles from Catharanthus Roseus Induces Caspase-Mediated Apoptosis in Cervical Cancer Cells (HeLa). Artificial Cells, Nanomedicine, and Biotechnology 47 (1): 1938-46.

Krishnaswamy, Haribabu, Sivaprakash Muthukrishnan, Sathish Thanikodi, Godwin Arockiaraj Antony, and Vijayan Venkatraman. (2020). Investigation of Air Conditioning Temperature Variation by Modifying the Structure of Passenger Car Using Computational Fluid Dynamics. Thermal Science 24 (1 Part B): 495-98.

Maheswari, T. N. Uma, Archana Venugopal, Nivedhita Malli Sureshbabu, and Prathiba Ramani. (2018). Salivary Micro RNA as a Potential Biomarker in Oral Potentially Malignant Disorders: A Systematic Review. Ci Ji Yi Xue Za Zhi = Tzu-Chi Medical Journal 30 (2): 55-60.

Malli Sureshbabu, Nivedhitha, Kathiravan Selvarasu, Jayanth Kumar V, Mahalakshmi Nandakumar, and Deepak Selvam. (2019). Concentrated Growth Factors as an Ingenious Biomaterial in Regeneration of Bony Defects after Periapical Surgery: A Report of Two Cases. Case Reports in Dentistry 2019 (January): 7046203.

Mathew, M. G., S. R. Samuel, A. J. Soni, and K. B. Roopa. (2020). Evaluation of Adhesion of Streptococcus Mutans, Plaque Accumulation on Zirconia and Stainless Steel Crowns, and Surrounding Gingival Inflammation in Primary. Clinical Oral Investigations. https://link.springer. com/article/10.1007/s00784-020-03204-9.

Mehta, Meenu, Deeksha, Devesh Tewari, Gaurav Gupta, Rajendra Awasthi, Harjeet Singh, Parijat Pandey, et al. (2019). Oligonucleotide Therapy: An Emerging Focus Area for Drug Delivery in Chronic Inflammatory Respiratory Diseases. Chemico-Biological Interactions 308 (August): 206-15.

Misra, Satya Ranjan, Y. Uday Shankar, Varun Rastogi, and G. Maragathavalli. (2015). Metastatic Hepatocellular Carcinoma in the Maxilla and Mandible, an Extremely Rare Presentation. Contemporary Clinical Dentistry 6 (Suppl 1): S117-21.

Muthukrishnan, A., and L. B. Kumar. (2017). Actinic Cheilosis: Early Intervention Prevents Malignant Transformation. Case Reports. https://casereports.bmj. com/content/2017/bcr-2016-218654.short.

Muthukrishnan, Arvind, Laliytha Bijai Kumar, and Gomathi Ramalingam. (2016). Medication-Related Osteonecrosis of the Jaw: A Dentist's Nightmare. BMJ Case Reports 2016 (April). https://doi.org/10.1136/bcr2016-214626.

Muthukrishnan, Arvind, and Saman Warnakulasuriya. (2018). Oral Health Consequences of Smokeless Tobacco Use. The Indian Journal of Medical Research 148 (1): 35-40.

Muthukrishnan, Sivaprakash, Haribabu Krishnaswamy,
Sathish Thanikodi, Dinesh Sundaresan, and Vijayan Venkatraman. (2020). Support Vector Machine for Modelling and Simulation of Heat Exchangers. Thermal Science 24 (1 Part B): 499-503.

Patil, Santosh R., G. Maragathavalli, Kazuyuki Araki, Ibrahim A. Al-Zoubi, Mohammed G. Sghaireen, Ravi Kumar Gudipaneni, and Mohammad Khursheed Alam. (2018). Three-Rooted Mandibular First Molars in a Saudi Arabian Population: A CBCT Study. Pesquisa Brasileira Em Odontopediatria E Clinica Integrada 18 (1): 4133.

Pattanaik, Seema, B. V. J. Vikas, Bikash Pattanaik, Shailendra Sahu, and Savita Lodam. (2010). "Denture Stomatitis: A Literature Review. Journal of Indian Academy of Oral Medicine and Radiology 22 (3): 136.

Pc, J., T. Marimuthu, and P. Devadoss. (2018). Prevalence and Measurement of Anterior Loop of the Mandibular Canal Using CBCT: A Cross Sectional Study. Clinical Implant Dentistry and Related Research. https:// europepmc.org/article/med/29624863.

Perić, Mirjana, Rade Živković, Aleksandra Milić Lemić, Milena Radunović, Biljana Miličić, and Valentina Arsić Arsenijević. (2018). The Severity of Denture Stomatitis as Related to Risk Factors and Different Candida Spp. Oral Surgery, Oral Medicine, Oral Pathology and Oral Radiology, March. https://doi.org/10.1016/j. oooo.2018.03.003.

Pesee, S., and T. Arpornsuwan. (2015). Salivary Cytokine Profile in Elders with Candida-related Denture Stomatitis. Gerodontology. https://onlinelibrary.wiley.com/doi/ abs/10.1111/ger.12064.

Ramadurai, Neeraja, Deepa Gurunathan, A. Victor Samuel, Emg Subramanian, and Steven J. L. Rodrigues. (2019). Effectiveness of 2\% Articaine as an Anesthetic Agent in Children: Randomized Controlled Trial. Clinical Oral Investigations 23 (9): 3543-50.

Ramesh, Asha, Sheeja Varghese, Nadathur D. Jayakumar, and Sankari Malaiappan. (2018). Comparative Estimation of Sulfiredoxin Levels between Chronic Periodontitis and Healthy Patients - A Case-Control Study. Journal of Periodontology 89 (10): 1241-48.

Rohini, S., and V. Jayanth Kumar. (2017). Incidence of Dental Caries and Pericoronitis Associated with Impacted Mandibular Third Molar-A Radiographic Study. Research Journal of Pharmacy and Technology 10 (4): 1081-84.

Samuel, Melvin S., Jayanta Bhattacharya, Sankalp Raj, Needhidasan Santhanam, Hemant Singh, and N. D. Pradeep Singh. (2019). Efficient Removal of Chromium (VI) from Aqueous Solution Using Chitosan Grafted Graphene Oxide (CS-GO) Nanocomposite. International Journal of Biological Macromolecules 121 (January): 285-92.

Samuel, Srinivasan Raj, Shashidhar Acharya, and Jeevika Chandrasekar Rao. (2020). School Interventions-Based 
Prevention of Early-Childhood Caries among 3-5-YearOld Children from Very Low Socioeconomic Status: Two-Year Randomized Trial. Journal of Public Health Dentistry 80 (1): 51-60.

Sathish, T., and S. Karthick. (2020). Wear Behaviour Analysis on Aluminium Alloy 7050 with Reinforced SiC through Taguchi Approach. Journal of Japan Research Institute for Advanced Copper-Base Materials and Technologies 9 (3): 3481-87.

Sharma, Parvarish, Meenu Mehta, Daljeet Singh Dhanjal, Simran Kaur, Gaurav Gupta, Harjeet Singh, Lakshmi Thangavelu, et al. (2019). Emerging Trends in the Novel Drug Delivery Approaches for the Treatment of Lung Cancer. Chemico-Biological Interactions 309 (August): 108720 .

Sridharan, Gokul, Pratibha Ramani, Sangeeta Patankar, and Rajagopalan Vijayaraghavan. 2(019). Evaluation of Salivary Metabolomics in Oral Leukoplakia and Oral Squamous Cell Carcinoma. Journal of Oral Pathology \& Medicine: Official Publication of the International Association of Oral Pathologists and the American Academy of Oral Pathology 48 (4): 299-306.

Steele, John C., Hadleigh J. Clark, Catherine H. L. Hong, Sabine Jurge, Arvind Muthukrishnan, A. Ross Kerr, David Wray, Linda Prescott-Clements, David H. Felix, and Thomas P. Sollecito. (2015). World Workshop on Oral Medicine VI: An International Validation Study of Clinical Competencies for Advanced Training in Oral Medicine. Oral Surgery, Oral Medicine, Oral Pathology and Oral Radiology 120 (2): 143-51. e7.

Subashri, A., and T. N. Maheshwari. (2016). Knowledge and Attitude of Oral Hygiene Practice among Dental Students. Research Journal of Pharmacy and Technology 9 (11): 1840-42.

Subha, M., and M. Arvind. (2019). Role of Magnetic Resonance Imaging in Evaluation of Trigeminal Neuralgia with Its Anatomical Correlation. Biomedical and Pharmacology Journal 12 (1): 289-96.

Thilakumara, I. P., and Jams Jayatilake. (2017). Denture-induced Stomatitis and Associated Factors in a
Group of Patients Attending a University Dental Hospital in Sri Lanka. Of Investigative and .... https://onlinelibrary. wiley.com/doi/abs/10.1111/jicd.12211.

Varghese, Sheeja Saji, Asha Ramesh, and Deepak Nallaswamy Veeraiyan. (2019). Blended Module-Based Teaching in Biostatistics and Research Methodology: A Retrospective Study with Postgraduate Dental Students. Journal of Dental Education 83 (4): 445-50.

Venugopal, Archana, and T. N. Uma Maheswari. (2016). Expression of Matrix Metalloproteinase-9 in Oral Potentially Malignant Disorders: A Systematic Review. Journal of Oral and Maxillofacial Pathology: JOMFP 20 (3): 474-79.

Venu, Harish, V. Dhana Raju, and Lingesan Subramani. (2019). Combined Effect of Influence of Nano Additives, Combustion Chamber Geometry and Injection Timing in a DI Diesel Engine Fuelled with Ternary (diesel-BiodieselEthanol) Blends. Energy 174 (May): 386-406.

Venu, Harish, Lingesan Subramani, and V. Dhana Raju. (2019). Emission Reduction in a DI Diesel Engine Using Exhaust Gas Recirculation (EGR) of Palm Biodiesel Blended with TiO2 Nano Additives. Renewable Energy 140 (September): 245-63.

Vignesh, R., Ditto Sharmin, C. Vishnu Rekha, Sankar Annamalai, and Parisa Norouzi Baghkomeh. (2019). Management of Complicated Crown-Root Fracture by Extra-Oral Fragment Reattachment and Intentional Reimplantation with 2 Years Review. Contemporary Clinical Dentistry 10 (2): 397-401.

Vijayakumar Jain, S., M. R. Muthusekhar, M. F. Baig, P. Senthilnathan, S. Loganathan, P. U. Abdul Wahab, M. Madhulakshmi, and Yogaen Vohra. (2019). Evaluation of Three-Dimensional Changes in Pharyngeal Airway Following Isolated Lefort One Osteotomy for the Correction of Vertical Maxillary Excess: A Prospective Study. Journal of Maxillofacial and Oral Surgery 18 (1): 139-46.

Vijayashree P, Jayaseelan. (2019). In Silico Validation of the Non-Antibiotic Drugs Acetaminophen and Ibuprofen as Antibacterial Agents against Red Complex Pathogens. Journal of Periodontology 90 (12): 1441-48. 